\title{
APLICAÇÃO DA TÉCNICA DE DISPERSÃo DA MATRIZ EM FASE SÓLIDA (DMFS) NA ANÁLISE DE PESTICIDAS EM QUIABO POR CG-EM
}

\author{
Haroldo Silveira Dórea* e Waneide Gomes Lopes \\ Departamento de Química, Universidade Federal de Sergipe, Av. Mal. Rondon, s/n, 49100-000 São Cristóvão - SE
}

Recebido em 19/9/03; aceito em 30/6/04; publicado na web em 8/10/04

\begin{abstract}
APPLICATION OF THE MATRIX SOLID PHASE DISPERSION (MSPD) TECHNIQUE IN THE ANALYSIS OF PESTICIDES IN OKRA BY GC-MS. A matrix solid phase dispersion and gas chromatography-mass selective detection method for the simultaneous determination of monocrotophos, methyl parathion, cypermethrin and deltamethrin in okra is described. Analyses of $2 \mathrm{~g}$ of fortified okra (0.05-0.75 mg kg-1) showed an average recovery of 96.2\% (71.4-128.4\%) and average relative standard deviation of $11.7 \%$ (1.4-37.1\%). The cypermethrin recovery at the lower level was above $130 \%$. The limit of detection ranged from 0.02 to $0.15 \mathrm{mg} \mathrm{kg}^{-1}$. The procedure was applied to the okra samples and has found $0.56 \mathrm{mg} \mathrm{kg}^{-1}$ of cypermethrin-cis, 0.75 $\mathrm{mg} \mathrm{kg}-1$ of cypermethrin-trans and $2.71 \mathrm{mg} \mathrm{kg}^{-1}$ of deltamethrin.
\end{abstract}

Keywords: MSPD; pesticides; GC-MS.

\section{INTRODUÇÃO}

O uso de pesticidas é ainda a principal estratégia no campo para o combate e a prevenção de pragas agrícolas. Esses compostos, porém, são potencialmente tóxicos ao homem, podendo causar efeitos adversos ao sistema nervoso central e periférico, ter ação imunodepressora, ser cancerígeno, entre outros males ${ }^{1}$.

O estudo de novos métodos para a análise multirresíduo de pesticidas em alimentos tem despertado interesse pelo fato de muitos laboratórios adotarem métodos que utilizam extração líquidolíquido $^{2,3}$ como rotina. Essa técnica, além de ser tediosa, morosa e de difícil automação, consome grandes quantidades de solventes orgânicos, linha contrária à tendência atual de se reduzir ao máximo os resíduos de solventes nos laboratórios.

A etapa de preparação da amostra torna-se um desafio para obtenção de métodos mais rápidos, que utilizem menores quantidades de solventes orgânicos, com maior sensibilidade e para matrizes com baixa concentração dos analitos. Extração com fluido supercrítico, EFS "Supercritical Fluid Extraction-SFE" 4,5 e extração em fase sólida, EFS "Solid Phase Extraction-SPE" são técnicas utilizadas para responder a essas exigências na extração de resíduos de pesticidas em alimentos. Outras técnicas, como "Solid-Phase MicroextractionSPME", "Accelerated Solvent Extraction-ASE", "Subcritical Water Extraction-SWE", "Pressurized Solvent Extraction-PSE", "Microwave Assisted Extraction-MAE", entre outras, estão sendo desenvolvidas ${ }^{7}$.

\section{Dispersão da Matriz em Fase Sólida (DMFS)}

Introduzida por Barker e colaboradores em $1989^{8}$, a dispersão da matriz em fase sólida, DMFS "Matrix Solid Phase DispersionMSPD”, resolveu muitos problemas de extração de matrizes sólidas e semi-sólidas, quando se utiliza uma fase sólida. A técnica combina diretamente a amostra com um adsorvente (dispersante), permitindo a realização simultânea de várias etapas no preparo da amostra.

A extração em fase sólida (EFS) na sua forma convencional re-

*e-mail: haroldo@ufs.br quer matrizes líquidas, relativamente não viscosas, livres de material particulado e no estado homogêneo 9 . Etapas anteriores são necessárias para preparar matrizes sólidas e semi-sólidas para introdução no cartucho de EFS e posterior purificação e/ou eluição. Os resultados têm mostrado que suas vantagens a tornam superior à extração líquido-líquido (ELL) e tem desempenho (em termos de recuperação, precisão, tempo e número de etapas) similar à extração com fluido supercrítico na análise de pesticidas em frutas ${ }^{10}$.

A técnica por DMFS consiste basicamente em introduzir a amostra em um recipiente contendo um suporte sólido (adsorvente), misturar até homogeneização, transferir o material (matriz e adsorvente) para a coluna e eluir com solvente apropriado. A coluna DMFS consiste, portanto, da matriz dispersa no adsorvente ${ }^{9}$.

O suporte sólido ou dispersante serve para várias funções. Primeiro atua como abrasivo, promovendo o rompimento da estrutura física da amostra; segundo, como adsorvente de compostos da matriz; terceiro, o material misturado pode ser empacotado na coluna (pressionando o êmbolo ajustado ao cartucho, como uma seringa, até a parte inferior do cartucho) e os analitos podem ser eluídos seqüencialmente com o eluente; quarto, a matriz distribuída no suporte produz um único material com a fase sólida da coluna, permitindo um novo grau de fracionamento da amostra?.

A escolha do suporte-adsorvente recai na polaridade do analito e na natureza da matriz ${ }^{9-11}$. Para aplicações que requerem uma fase lipofílica quimicamente ligada "bonded phase", geralmente o suporte-adsorvente faz uso de materiais como $\mathrm{C}_{18}$ e $\mathrm{C}_{8}$. O tipo de ligante $\left(\mathrm{C}_{8}\right.$ e $\left.\mathrm{C}_{18}\right)$, a quantidade e a porcentagem também afetarão os resultados, portanto, deve-se examinar as fases disponíveis para cada aplicação. $\mathrm{O}$ isolamento de analitos mais polares é realizado com suportes sólidos polares e o de analitos menos polares com suportes menos polares.

As interações dos componentes do sistema por dispersão da matriz em FS envolvem o analito com o suporte sólido, com a fase quimicamente ligada $\left(\mathrm{C}_{18}\right)$ e com a matriz dispersa, a matriz com o suporte sólido e com a fase quimicamente ligada, todos os componentes acima interagindo com o solvente de eluição e as interações dinâmicas de todos os componentes ocorrendo simultaneamente ${ }^{9}$.

Na etapa de homogeneização da amostra com o adsorvente, podese fazer a adição de reagentes para modificar a amostra (modifica- 
dores), como antioxidantes, agentes quelantes, ácidos, bases etc. Estes procedimentos podem ser realizados para afetar a seqüência da eluição, distribuição e/ou retenção do analito. Ocorrem modificações na matriz no momento da mistura. A ionização ou supressão da ionização dos analitos e dos componentes da amostra pode afetar grandemente a natureza das interações entre eles e com o solvente de eluição?.

O condicionamento e a pré-lavagem da mistura matriz-suporte na coluna DMFS também podem ser usados para a ativação do adsorvente e eliminação de interferentes ${ }^{10}$.

A natureza da coluna DMFS e a faixa de interações permitem o isolamento de analitos de polaridades diferentes ou de uma classe de substâncias, em um único solvente ou em diferentes solventes de eluição. Este isolamento torna a DMFS uma técnica apropriada para isolar e/ou purificar multirresíduos a partir de matrizes complexas ${ }^{10,11}$.

Assim como na extração em fase sólida, o suporte sólido, a fase quimicamente ligada e o solvente de eluição são parâmetros críticos, mas esses certamente têm influência menor que a dispersão da matriz do topo até a base da coluna DMFS. Na extração em FS, a maioria das substâncias da amostra é retida nos primeiros milímetros do topo da coluna (efeito da matriz), enquanto que em DMFS a deposição da amostra com o adsorvente na coluna é uniforme por toda sua extensão?.

Em alguns casos o eluato da coluna DMFS está satisfatoriamente limpo para ser injetado diretamente no cromatógrafo, porém na maioria das vezes os interferentes devem ser removidos com uma purificação adicional, conectando-se em série uma segunda coluna (co-coluna) contendo outra fase sólida na coluna DMFS, ou colocando-se uma FS (p.ex. alumina, Florisil, sílica, $\mathrm{C}_{8}, \mathrm{C}_{18}$ ) na coluna DMFS antes de adicionar a mistura matriz-suporte ${ }^{10,11}$.

Neste trabalho é desenvolvido um método por dispersão da matriz em fase sólida para analisar os pesticidas organofosforados, monocrotofós e paration metílico, e os piretróides sintéticos, cipermetrina e deltametrina, em amostras de quiabo. Este alimento foi escolhido por ser o principal produto cultivado no Perímetro Irrigado Califórnia, localizado no semi-árido sergipano, e um importante componente da alimentação da população regional ${ }^{12}$.

\section{PARTE EXPERIMENTAL}

\section{Reagentes e soluções}

Acetato de etila (Merck, Darmstadt, Germany), óxido de alumínio neutro (Merck, Darmstadt, Germany) 70-290 mesh, sílica gel 70-230 mesh (Merck, Darmstadt, Germany) e sílica gel 65 mesh (produzida na UFS). Padrões dos pesticidas monocrotofós (Riedelde-Haën, Seelze, 99\%), paration metílico (Institute of Organic Industrial Chemistry, Warsaw, Poland, 99,4\%), cipermetrina (AccuStandard, New Haven, USA, 99\%) e deltametrina (AccuStandard, New Haven, USA, 99,6\%). A Figura 1 mostra as estruturas químicas dos pesticidas estudados. As soluções padrão estoques foram preparadas com concentrações de $200 \mu \mathrm{g} \mathrm{mL} \mathrm{m}^{-1} \mathrm{em}$ acetato de etila. A partir destas foram preparadas soluções padrão de trabalho com concentrações diferenciadas, segundo a sensibilidade do composto no detector de espectrometria de massas: paration

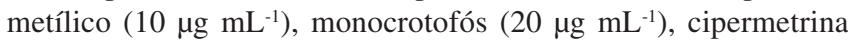
$\left(30 \mu \mathrm{g} \mathrm{mL} L^{-1}\right)$ e deltametrina $\left(30 \mu \mathrm{g} \mathrm{mL}^{-1}\right)$.

\section{Preparação da amostra}

Amostras de quiabo não contaminadas, adquiridas com microprodutores que não utilizam pesticidas, foram utilizadas para desenvolver a metodologia por DMFS. Para o teste final foram coletadas<smiles>CNC(=O)C=C(C)OP(=O)(OC)OC</smiles><smiles>CC(C)=CC1C(C(=O)O)C1(C)C</smiles>

c

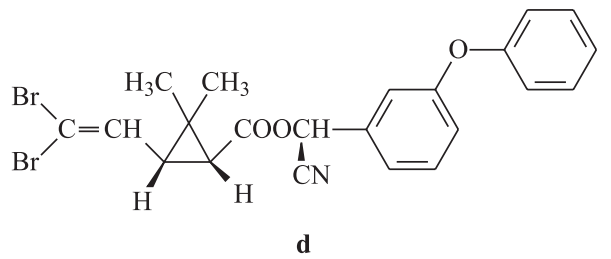

Figura 1. Estrutura química dos pesticidas (a) monocrotofós, (b) paration metílico, (c) cipermetrina e (d) deltametrina

amostras comerciais no Mercado de Aracaju, em abril de 2002, com procedência de Canindé do São Francisco.

Após o recebimento das amostras, as mesmas foram condicionadas em sacos plásticos, vedadas, identificadas e conservadas em freezer. No momento da extração as amostras foram retiradas e deixadas à temperatura ambiente até descongelarem. Em seguida, os quiabos foram cortados em pequenos pedaços, incluindo-se a cabeça do quiabo, pesados $2 \mathrm{~g}$, adicionados $0,5 \mathrm{~mL}$ da solução padrão de trabalho contendo os pesticidas e $4 \mathrm{~g}$ de sílica gel (70-230 mesh). Após a homogeneização da mistura em almofariz por $2 \mathrm{~min}$, o conteúdo foi transferido para a coluna de polietileno $(20 \mathrm{~mL})$ contendo $1 \mathrm{~cm}$ de alumina neutra, $\mathrm{Al}_{2} \mathrm{O}_{3}$, (co-coluna) e lã de vidro como "base de sustentação". Os pesticidas foram eluídos com $40 \mathrm{~mL}$ de acetato de etila e o eluato foi concentrado em rota-evaporador até o volume final de aproximadamente $5 \mathrm{~mL}$. O extrato foi mantido em freezer até o momento da análise por CG-EM.

\section{Condições cromatográficas}

O CG-EM da Shimadzu, modelo QP5050A, Kyoto, Japão, foi utilizado nas análises dos pesticidas em quiabo. Equipado com injetor com e sem divisão de fluxo, a $250^{\circ} \mathrm{C}$, e coluna DB-5 (30 m x 0,25 mm $\mathrm{x} 0,25 \mu \mathrm{m})$. Forno: temperatura inicial, $160^{\circ} \mathrm{C}(1 \mathrm{~min})$; taxa de aquecimento de $10^{\circ} \mathrm{C} / \mathrm{min}$ até $250{ }^{\circ} \mathrm{C}(5 \mathrm{~min})$. Temperatura da interface, $280{ }^{\circ} \mathrm{C}$. Gás de arraste, hélio (70 kPa). Volume de injeção: $1 \mu \mathrm{L}$ de solução padrão ou extrato da amostra. Razão do divisor, 1:10. O espectrômetro de massas foi operado no modo MIS (monitoramento de íons selecionados). Modo de ionização: impacto de elétrons a $70 \mathrm{eV}$. Fragmentos selecionados para os pesticidas estudados: $\mathrm{m} / \mathrm{z}$ 125, 127, 163, 181, 209, 253 e 263.

\section{RESULTADOS E DISCUSSÃO}

A escolha dos pesticidas baseou-se em um estudo realizado em área de irrigação do semi-árido nordestino, onde cerca de 8.000 t/ano são produzidos pela cultura de quiabo $(57 \%$ da produção total da 
Tabela 1. Tempo de retenção, fórmula química, massa molar, íons selecionados, equação da reta e coeficiente de correlação das curvas analíticas dos pesticidas estudados

\begin{tabular}{lcccccc}
\hline Pesticida & $\mathrm{t}_{\mathrm{R}}(\mathrm{min})$ & Fórmula química & $\mathrm{M}\left(\mathrm{g} \mathrm{mol}{ }^{-1}\right)$ & $\mathrm{MIS}(\mathrm{m} / \mathrm{z})^{*}$ & Equação da Reta $\mathrm{y}=\mathrm{bx}+\mathrm{a}$ & $\mathrm{r}$ \\
\hline Monocrotofós & 5,2 & $\mathrm{C}_{7} \mathrm{H}_{14} \mathrm{O}_{5} \mathrm{NP}$ & 223 & 127 & $5,510^{4} \mathrm{x}-2,110^{4}$ & 0,999 \\
Paration metílico & 7,0 & $\mathrm{C}_{8} \mathrm{H}_{10} \mathrm{NO}_{5} \mathrm{PS}$ & 263 & 125,263 & $4,010^{4} \mathrm{x}-5,210^{3}$ & 0,999 \\
Cipermetrina-cis & 12,6 & $\mathrm{C}_{22} \mathrm{H}_{19} \mathrm{C}_{12} \mathrm{NO}_{3}$ & 415 & 163,181 & $1,510^{4} \mathrm{x}-9,910^{3}$ & 0,999 \\
Cipermetrina-trans & 12,8 & $\mathrm{C}_{22} \mathrm{H}_{19} \mathrm{C}_{12} \mathrm{NO}_{3}$ & 415 & 163,181 & $1,310^{4} \mathrm{x}-9,610^{3}$ & 0,997 \\
Deltametrina & 14,7 & $\mathrm{C}_{22} \mathrm{H}_{19} \mathrm{BR}_{2} \mathrm{NO}_{3}$ & 503 & 209,253 & $1,210^{4} \mathrm{x}-4,310^{3}$ & 0,999 \\
\hline
\end{tabular}

* íons usados para quantificação

área) ${ }^{12}$. Em seguida, foram avaliadas as condições cromatográficas (CG-EM) através da injeção de soluções padrão dos pesticidas, inicialmente no modo de aquisição de varredura linear ("Scan"). Os íons diagnósticos selecionados para o monitoramento dos analitos encontram-se reunidos na Tabela 1. Os resultados quantitativos foram obtidos no modo MIS. Os isômeros cis e trans do piretróide cipermetrina foram analisados individualmente, como dois compostos diferentes.

A quantificação foi feita empregando-se a padronização externa. A curva analítica foi obtida pelo método dos mínimos quadrados para o intervalo de concentração de 0,5 a $6,0 \mu \mathrm{g} \mathrm{mL}^{-1}$ para os pesticidas analisados; os valores do coeficiente de correlação e a equação da reta para cada pesticida são mostrados na Tabela 1. Os valores do coeficiente de correlação ( $r>0,997)$ indicam que o modelo linear pode ser usado na quantificação dos analitos.

Os padrões de pesticidas foram adicionados às amostras de quiabo não contaminadas antes da adição do dispersante (adsorvente) e esperou-se cerca 30 min para que houvesse maior interação dos pesticidas com a amostra. Devido à pequena quantidade de amostra utilizada (2 g), é necessária a adição de um volume reduzido da solução padrão de trabalho contendo os pesticidas, para garantir a penetração de todos os pesticidas na matriz. Um volume excessivo da solução padrão poderia ocasionar em perda dos pesticidas, ficando parte desses no recipiente onde haverá a homogeneização.

Foram realizados testes com os adsorventes sílica e alumina neutra em várias proporções. Foram feitos também testes com e sem cocoluna. Os melhores resultados de recuperação foram obtidos utilizando-se sílica gel (70-230 mesh) como dispersante e alumina neutra na co-coluna (Figura 2). Devido aos bons resultados obtidos em trabalhos anteriores ${ }^{11,13,14}$, acetato de etila foi usado como eluente.

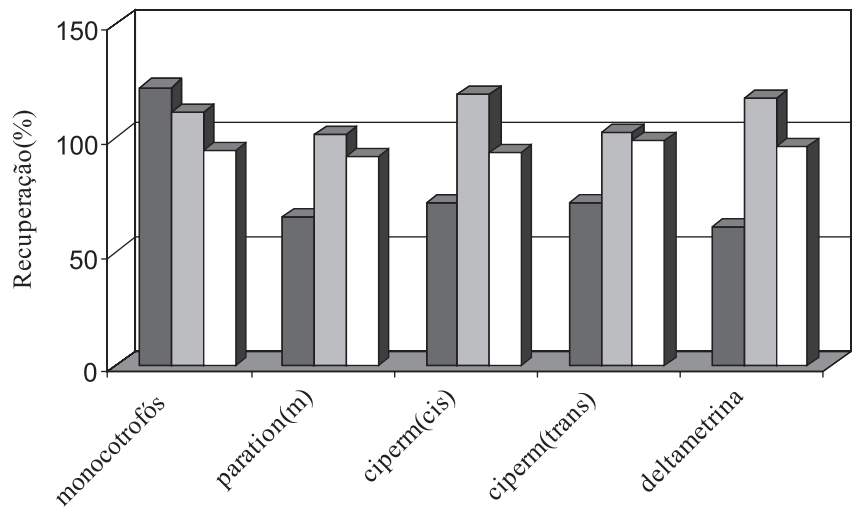

$\square$ sílica (65 mesh) $\square$ sílica (70-230 mesh) $\square$ sílica (70-230 mesh)/alumina

Figura 2. Recuperação $(n=3)$ dos pesticidas em amostras de quiabo fortificadas com monocrotofós $\left(0,5 \mathrm{mg} \mathrm{kg}^{-1}\right)$, paration metílico $\left(0,25 \mathrm{mg} \mathrm{kg}^{-1}\right)$, cipermetrina cis elou trans $\left(0,75 \mathrm{mg} \mathrm{kg}^{-1}\right)$ e deltametrina $\left(0,75 \mathrm{mg} \mathrm{kg}^{-1}\right)$
A Figura 2 mostra os resultados de recuperação para os três melhores procedimentos, com fortificação de 0,25 a $0,75 \mathrm{mg} \mathrm{kg}^{-1}$ (nível mais alto de fortificação) em triplicata, para os pesticidas estudados. De um modo geral, os menores resultados de recuperação foram obtidos tendo sílica gel (65 mesh) como dispersante e sem co-coluna, com média de recuperação de 78,2\% (média da estimativa do desvio padrão relativo, DPR, de 15,8\%) para todos os pesticidas. Substituindo-se o dispersante pela sílica gel (70-230 mesh), de outra marca, e também sem co-coluna, a recuperação média foi de 110,1\%. Os cromatogramas mostraram presença de interferentes, o que explica a recuperação superior a $100 \%$. Além disto, a precisão estimada pelo DPR (32,7\%) foi inferior à obtida com sílica gel (65 mesh). A combinação entre sílica gel como dispersante e alumina neutra como fase sólida da co-coluna foi a que forneceu melhores resultados. A recuperação média foi de 95,7\% (DPR de 6,6\%).

A alumina neutra também se mostrou satisfatória como co-coluna na determinação de 11 pesticidas em matrizes de plantas medicinais, obtendo-se resultados de recuperação entre 70,6 e 116,7\% ${ }^{15}$.

Após a otimização inicial, níveis de fortificação mais baixos foram estudados. A Tabela 2 mostra os resultados de recuperação em 3 níveis de concentrações diferentes e a estimativa de DPR (\%) dos pesticidas selecionados nas amostras de quiabo pelo método de extração por DMFS, usando sílica gel (70-230 mesh) como dispersante e alumina neutra como fase sólida da co-coluna. As amostras de quiabo foram fortificadas entre 0,05 e $0,75 \mathrm{mg} \mathrm{kg}^{-1}$ e basicamente todos os resultados apresentaram valores de recuperação $(n=3)$ entre 71,4 e $128,4 \%$, dando uma recuperação média de $96,2 \%$. Apenas a

Tabela 2. Resultados de recuperação $(n=3)$ e estimativa de desvio padrão relativo, em 3 níveis de fortificação, dos pesticidas estudados em amostras de quiabo extraídas com o método proposto por DMFS (sílica, co-coluna)

\begin{tabular}{lccr}
\hline Pesticidas & $\begin{array}{c}\text { Fortificação } \\
\left(\mathrm{mg} \mathrm{kg}^{-1}\right)\end{array}$ & $\begin{array}{c}\text { Recuperação } \\
\text { média }(\%)\end{array}$ & $\begin{array}{r}\text { DPR* } \\
(\%)\end{array}$ \\
\hline Monocrotofós & 0,50 & 98,7 & 4,2 \\
& 0,25 & 73,0 & 23,9 \\
Paration metílico & 0,10 & 126,2 & 1,4 \\
& 0,25 & 91,4 & 5,0 \\
Cipermetrina-cis & 0,15 & 75,1 & 21,2 \\
& 0,05 & 112,9 & 2,8 \\
Cipermetrina-trans & 0,75 & 93,3 & 6,2 \\
& 0,40 & 91,4 & 17,2 \\
Deltametrina & 0,15 & 179,8 & 7,3 \\
& 0,75 & 98,9 & 7,6 \\
& 0,40 & 93,9 & 14,6 \\
& 0,15 & 184,4 & 5,1 \\
& 0,75 & 96,1 & 10,0 \\
& 0,40 & 71,4 & 37,1 \\
& 0,15 & 128,4 & 11,5 \\
\hline
\end{tabular}

* Estimativa do desvio padrão relativo, $D P R(\%)=\frac{S D .100}{\bar{x}}$ 
cipermetrina (cis e trans) apresentou resultados de recuperação não satisfatórios $(>130 \%)$ para o nível de fortificação mais baixo. Isso pode ser justificado por se tratar do limite de detecção do pesticida no CG/EM e pela presença de compostos endógenos da matriz.

Os limites de detecção (LD) e quantificação (LQ) foram estabelecidos como 3 e 10 vezes a razão sinal/ruído (s/n), respectivamente, nas condições cromatográficas estabelecidas e levando-se em consideração a quantidade da sub-amostra utilizada no método e do volume do extrato. Os resultados mostraram que houve uma variação entre 0,02 e $0,15 \mathrm{mg} \mathrm{kg}^{-1}$ para LD e 0,1 a $0,7 \mathrm{mg} \mathrm{kg}^{-1}$ para LQ. O paration metílico mostrou maior sensibilidade que os demais pesticidas.

O Limite Máximo de Resíduos (LMR) é a concentração máxima de resíduos de um pesticida para que se permita o uso legal, tanto na superfície quanto na parte interna dos produtos alimentícios para consumo humano ou de animais ${ }^{16}$. Comparando os valores de LD com relação ao LMR estabelecido pelo $\operatorname{Codex}^{16}$ (Tabela 3), observase que os valores de LD obtidos pelo método proposto estão abaixo do exigido para esse tipo de alimento.

Comparando o método proposto com outros métodos que usaram DMFS para analisar pesticidas em alimentos (Tabela 4), observa-se uma variação na quantidade de amostras da ordem de 10 vezes (0,5 a $5 \mathrm{~g})$; já a relação amostra/suporte tem sido definida experimentalmente, pois pode ter valores de $1 / 1 ; 1 / 0,5 ; 1 / 1,6$ e $1 / 2$. Quanto à relação volume do eluente/coluna DMFS (composta de amostra +

Tabela 3. Limites de detecção (LD) e de quantificação (LQ) dos pesticidas estudados, em comparação com os limites máximos de resíduos (LMR)

\begin{tabular}{lccc}
\hline Pesticida & $\begin{array}{c}\mathrm{LD} \\
\left(\mathrm{mg} \mathrm{kg}^{-1}\right)\end{array}$ & $\begin{array}{c}\mathrm{LQ} \\
\left(\mathrm{mg} \mathrm{kg}^{-1}\right)\end{array}$ & $\begin{array}{c}\mathrm{LMR}^{16 *} \\
\left(\mathrm{mg} \mathrm{kg}^{-1}\right)\end{array}$ \\
\hline Monocrotofós & 0,10 & 0,5 & 0,2 \\
Paration metílico & 0,02 & 0,1 & 0,2 \\
Cipermetrina & 0,15 & 0,7 & 1,0 \\
Deltametrina & 0,15 & 0,7 & 0,2 \\
\hline
\end{tabular}

* Para produtos alimentícios semelhantes ao quiabo (hortaliças). suporte + fase sólida da co-coluna) tem variado entre 1/4; $1 / 5 ; 1 / 8$ e 1/10 (Tabela 4).

O fragmentograma apresentado na Figura 3 mostra a comparação entre uma solução padrão, uma amostra de quiabo fortificada com os pesticidas estudados (de 0,25 a $0,75 \mathrm{mg} \mathrm{kg}^{-1}$ ) e o branco. $\mathrm{O}$ aumento na linha de base após 10 min de análise foi devido ao íon $\mathrm{m} / \mathrm{z}$ 253, selecionado para a quantificação da deltametrina.

\section{Aplicação em amostra comercial}

Os resultados para as amostras comerciais foram obtidos corrigindo-se os valores de recuperação do método proposto com a média de recuperação para os três níveis de fortificação. As amostras foram
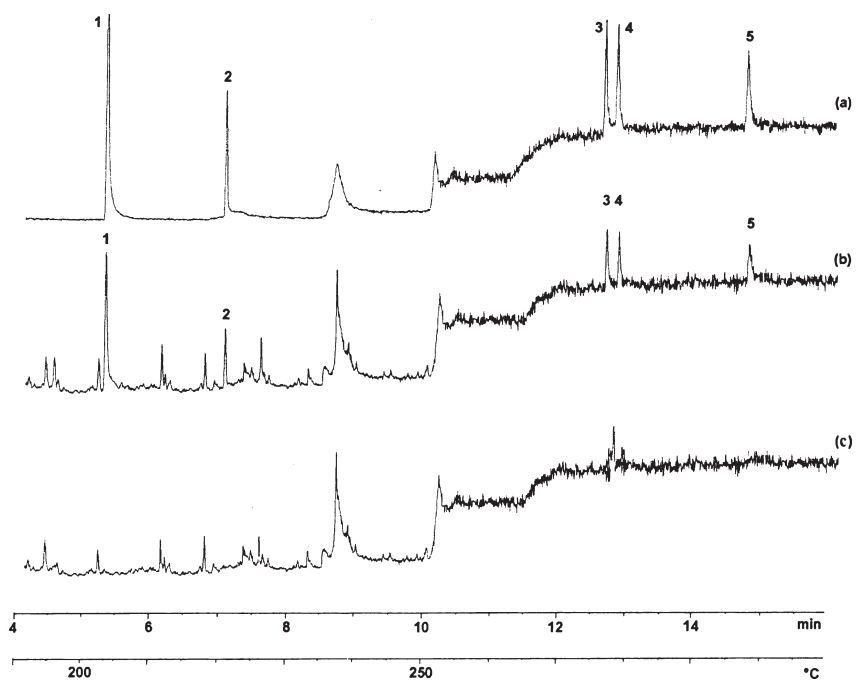

Figura 3. CG-EM (MIS), fragmentogramas obtidos das análises: (a) da solução padrão de trabalho contendo os pesticidas estudados, (b) da amostra de quiabo fortificada pelo método proposto por DMFS (nível 3, mais concentrado, ver Tabela 2) e (c) do branco. Identificação dos pesticidas: 1monocrotofós, 2-paration metílico, 3-cipermetrina-cis, 4-cipermetrina-trans e 5-deltametrina

Tabela 4. Dados da literatura sobre as aplicações de DMFS na análise de resíduos de pesticidas em alimentos

\begin{tabular}{|c|c|c|c|c|c|c|c|}
\hline \multirow[b]{2}{*}{ Parâmetros avaliados } & \multicolumn{6}{|c|}{ REFERÊNCIAS } & \multirow[b]{2}{*}{$\begin{array}{c}\text { Proposto } \\
\text { (parâmetros } \\
\text { selecionados) }\end{array}$} \\
\hline & 11 & 13 & 14 & 17 & 18 & 19 & \\
\hline Amostra & Frutas & $\begin{array}{l}\text { Frutas, } \\
\text { vegetais }\end{array}$ & $\begin{array}{c}\text { Frutas, } \\
\text { vegetais }\end{array}$ & Vegetais & Frutas & $\begin{array}{l}\text { Frutas, } \\
\text { vegetais }\end{array}$ & Quiabo \\
\hline Quantidade de amostra (g) & 1,0 & 0,5 & 0,5 & 5,0 & 0,1 & 0,5 & 2 \\
\hline Suporte & Florisil & $\mathrm{C}_{18}$ & $\mathrm{C}_{18}$ & Florisil & $\mathrm{C}_{8}$ & $\mathrm{C}_{8}$ & Sílica gel \\
\hline Quantidade de suporte (g) & 0,5 & 0,5 & 0,5 & 8,0 & 0,1 & 0,5 & 4 \\
\hline Co-coluna & $\begin{array}{l}\text { Sílica gel/ } \\
\text { sulfato de } \\
\text { sódio anidro }\end{array}$ & Sílica & Sílica & $\begin{array}{l}\text { Florisil/ } \\
\text { sulfato de } \\
\text { sódio anidro }\end{array}$ & Sílica & - & $\begin{array}{l}\text { Alumina } \\
\text { neutra }\end{array}$ \\
\hline $\begin{array}{l}\text { Quantidade de fase sólida } \\
\text { na co-coluna }(\mathrm{g})\end{array}$ & $0,5 / 3,0$ & 1,5 & 1,0 & $0,5 / 1 \mathrm{~cm}$ & não informado & - & $1 \mathrm{~cm}$ \\
\hline Eluente & $\begin{array}{l}\text { Acetato } \\
\text { de etila }\end{array}$ & $\begin{array}{l}\text { Acetato } \\
\text { de etila }\end{array}$ & $\begin{array}{l}\text { Acetato } \\
\text { de etila }\end{array}$ & $\begin{array}{c}\text { n-hexano: } \\
\text { acetona }(9: 1)\end{array}$ & $\begin{array}{c}\text { n-hexano, } \\
\text { acetato de etila }\end{array}$ & $\begin{array}{l}\text { Cloreto de } \\
\text { metileno }\end{array}$ & $\begin{array}{l}\text { Acetato } \\
\text { de etila }\end{array}$ \\
\hline Volume eluente (mL) & 40 & 10 & 10 & 60 & não informado & 10 & 40 \\
\hline
\end{tabular}


coletadas no mercado de Aracaju e analisadas em triplicata. Os pesticidas determinados foram cipermetrina-cis $\left(0,56 \mathrm{mg} \mathrm{kg}^{-1}\right)$, cipermetrina-trans $\left(0,75 \mathrm{mg} \mathrm{kg}^{-1}\right)$ e deltametrina $\left(2,71 \mathrm{mg} \mathrm{kg}^{-1}\right)$. A concentração da deltametrina encontra-se 13 vezes acima do LMR (Tabela 3).

\section{CONCLUSÃO}

O método proposto por dispersão da matriz em fase sólida (DMFS) mostrou-se eficiente para analisar os pesticidas monocrotofós, paration metílico, cipermetrina e deltametrina em amostras de quiabo, uma matriz com características diferentes das demais aplicadas com essa técnica. As amostras comerciais de quiabo mostraram valores acima do permitido pelo CODEX para deltametrina. Pequena quantidade de amostra, pouco consumo de solvente orgânico, poucas etapas envolvidas, sem manipulações químicas complicadas da amostra, conferem a essa técnica um ganho em termos de tempo, consumo de solvente e uma utilização mínima de materiais, tornando-a atrativa quando comparada com as técnicas clássicas de extração de pesticidas.

\section{AGRADECIMENTOS}

Os autores agradecem o apoio financeiro da FINEP/CTPETRO na aquisição do CG-EM e ao CNPq pela bolsa recebida por W. G. Lopes.

\section{REFERÊNCIAS}

1. Caldas, E. C.; Souza, L. C. K. R.; Rev. Saúde Pública 2000, 34, 5.

2. Luke, M. A.; Froberg, J. E.; Masumoto, H. T.; J. AOAC 1975, 58, 1020.

3. Pang, G. F.; Fan, C. L.; Chao, Y. Z.; J. AOAC Int. 1994, 77, 738.

4. Lanças, F. M.; Rissato, S. R.; J. Microcol. Sep. 1998, 10, 473.

5. Lanças, F. M.; Dórea, H. S.; Muller, R.; Fagundes, C.; Ciênc. Tecnol. Aliment. 1997, 17, 432.

6. Dórea, H. S.; Tadeo, J. L.; Sanchez-Brunete, C.; Chromatographia 1996, $43,380$.

7. Lanças, F. M.; J. Braz. Chem. Soc. 2003, 14, 183.

8. Barker, S. A.; Long, A. R.; Short, C. R.; J. Chromatogr, A 1989, 475, 353.

9. Barker, S. A.; LC-GC 1998, supplement, S37.

10. Dórea, H. S; Tese de Doutorado, Universidade de São Paulo, Brasil, 1999.

11. Dórea, H. S; Lanças, F. M.; J. Microcol. Sep. 1999, 11, 367.

12. Pinheiro, A. S.; Dórea, H. S.; Curituba 2001, 4, 81.

13. Torres,C. M.; Picó, Y.; Redondo, M. J.; Mañes, J.; J. Chromatogr., A 1996, $719,95$.

14. Navarro, M.; Picó, Y.; Marín, R.; Mañes, J.; J. Chromatogr., A 2002, 968 , 201.

15. Zuin, V. G.; Yariwake, J. H.; Lanças, F. M.; J. Braz. Chem. Soc. 2003, 14, 304.

16. FAO/OMS; Codex Alimentarius - Residuos de Plaguicidas em los alimentos, Roma, 1994, vol. 2.

17. Ling, Y. C.; Huang, I. P.; J. Chromatogr., A 1995, 695, 75.

18. Kristenson, E. M.; Haverkate, E. G. J.; Slooten, C. J.; Ramos, I.; Vreuls, R. J. J.; Brinkman, U. A. T.; J. Chromatogr., A 2001, 917, 277.

19. Blasco, C.; Picó, Y.; Manes, J.; Font, G.; J. Chromatogr., A 2002, 947, 227. 